\title{
Bacillus spp.; perspectiva de su efecto biocontrolador mediante antibiosis en cultivos afectados por fitopatógenos
}

\author{
Cristian Layton', Edna Maldonado', Luisa Monroy' \\ Lucia Constanza Corrales², Ligia Consuelo Sánchez²
}

\author{
1. Estudiantes de Bacteriología \\ 2. Docentes investigadoras Universidad Colegio Mayor de Cundinamarca. Bogotá, D.C.
}

Recibido: 01/09/2011 Aceptado: 10/11/2011

Correspondencia: Icorralesr07@gmail.com

\begin{abstract}
Resumen
El presente estudio documental evalúa el efecto biocontrolador del género Bacillus sp contra hongos fitopatógenos de plantas, particularmente, a través de relaciones antagónicas inductoras de muerte celular en términos inminentemente naturales. Fusarium oxysporum se encuentra muy relacionado con casos de marchitez vascular y pudrición de raíz en variedad de plantas, obstrucción de los vasos que permiten la circulación vegetal hasta causar amarillamiento de las hojas por imposibilidad en el transporte de nutrientes, causal de grandes pérdidas económicas en el campo agrícola nacional. Se han establecido varios mecanismos para controlar este hongo micelial dentro de las que se encuentran el uso extensivo y variable de agroquímicos y pesticidas, práctica que por sus efectos nocivos con el medio ambiente se ha comenzado a reemplazar por empleo de especies del género Bacillus. La acción biocontroladora de este género bacteriano esta mediada por su perfil bioquímico ya que son productores de múltiples metabolitos biológicamente activos, en el caso de Bacillus subtilis de Iturin A y fengycin y en Bacillus brevis de gramicidina S ( I-5) son capaces de inhibir el desarrollo y crecimiento normal de otros microorganismos, lo que sugiere su utilización para el biocontrol de plagas en aras al fortalecimiento de los actuales estándares de calidad en los procesos ambientales.
\end{abstract}

Palabras Clave: Bacillus sp, Fusarium oxysporum, biocontrolador, genes, metabolitos.

\section{Abstract \\ Bacillus spp.; perspective biocontrol through antibiosis effect on crops affected by phytopathogenics}

This desktop study evaluates the effect of the genus Bacillus sp biocontrol against plant pathogenic fungi, particularly by inducing antagonistic relations of cell death in terms natural imminently. Fusarium oxysporum is closely related to cases of vascular wilt androot rot in a variety of plants, obstruction of the vessels that circulate plant to cause yellowing of leaves due to the impossibility in the transport of nutrients, major cause of economic losses national agricultural field. Several mechanisms have been established to control this fungus mycelium within which are variable and extensive use of agrochemicals and pesticides, a practice which by its harmful 
effects to the environmenthas begun to replace the use of Bacillus spp. The action of this bacterial genus biocontrol is mediated by its biochemical profile since they are producing multiple biologically active metabolites in the case of Bacillus subtilis fengycin Iturin A and Bacillus brevis and gramicidin S (I-5) are capable of inhibiting development and normal growth of other microorganisms, suggesting its use for biocontrol of pests in order tostrengthen the existing quality standards in environmental processes.

Keywords: Bacillus sp, Fusarium oxysporum, biocontrol, genes, metabolites.

\section{Adaptación celular: un proceso en constante cambio}

Desde la década de los cuarenta se han descrito los mecanismos en los que se interrelacionan varios tipos de microorganismos caracterizados por propiedades intrínsecas variables pero que tienen como común denominador la supervivencia y la sostenibilidad de su especie, bien sea garantizándola a través de la competencia con otros microorganismos por nutrientes comunes ó mediante procesos de adaptación en relaciones intra e interespecificas $y$ en caso contrario en interacciones donde hay predominio de uno de esos microorganismos y la desaparición de los otros en un ambiente especifico: denominado relaciones antagónicas. Hecho que ha llevado a profundizar en su estudio para facilitar el proceso de eliminación de microorganismos que no son deseables bajo el principio de control de plagas y fitopatógenos con otros agentes biológicos. $(6,7,8)$.

Por otra parte, la estructura morfológica de la célula bacteriana ha sido estudiada a lo largo de la historia desde diversas perspectivas que han establecido su indudable funcionalidad en el mantenimiento de los procesos metabólicos y de comunicación celular así como de las funciones vitales de nutrición, respiración celular y expresión génica. En este orden de ideas, el continuo análisis de los cambios celulares que surgen durante los procesos de adaptación a otros microambientes ha llevado a dimensionar los mecanismos a través de los cuales los canales mecano sensitivos representan un rol fundamental en la preservación de la ultraestructura bacteriana, por ejemplo cuando se desarrollan dos microorganismos con acción antagónica en un mismo ambiente (9).
Estudios experimentales recientes indican dos nuevos elementos que han sido adicionados como mecanismos de adaptación, uno de los cuales es universal y otro de distribución más limitada. El primero fue el descubrimiento de los canales mecano sensitivos que abren rápidamente produciendo largas cavidades en la membrana citoplasmática que en respuesta incrementa la tensión en la membrana celular y el segundo el concepto de la pared celular como una estructura dinámica, la cual sufre cambios durante la adaptación al estrés (9).

Ahora bien, se puede partir de los cambios que surgen a nivel de adaptación para entender los procesos que debe asimilar la célula bacteriana desde el estimulo y reconocimiento de otro microorganismo hasta las acciones ejecutadas según sea el caso y que en este específicamente representa el censo a través de señales propias del sistema de comunicación celular bacteriana que activan los factores de expresión génica y lo que representa- transcripción, replicación y traducción de señales que finalmente van a generar la producción de polipéptidos con acción antimicrobiana (9).

\section{Bacillus spp: generalidades}

El género Bacillus, pertenece a la familia Bacillaceae, una de las familias bacterianas con mayor actividad bioquímica referenciada en la literatura científica que abarca tanto su utilización dentro de las actuales políticas de control biológico como el uso de los productos de su metabolismo para la industria.

Son bacilos aerobios y anaerobios facultativos, gram positivos, producen endosporas con morfología oval o cilíndrica que le permite resistir condiciones desfavorables en el ambiente, son móviles por la 
presencia de flagelos laterales, son catalasa positiva, presentando hemólisis variable y un crecimiento activo en un rango de $\mathrm{pH}$ entre 5.5 - 8.5. La propagación activa del microorganismo se produce en medios que presentan superficie húmeda. Las células en crecimiento no se propagan fácilmente en medios líquidos. Estos microorganismos por lo general crecen bien en agar sangre, produciendo colonias blanquecinas, grandes, extendidas e irregulares.

Dentro de las especies más representativas de este género con propiedades de antagonismo celular contra fitopatógenos encontramos a Bacillus brevis y Bacillus subtilis.

El metabolismo de B. subtilis es predominantemente respiratorio, siendo el oxígeno el aceptor terminal de electrones, por tanto en presencia de oxigeno resulta abundante crecimiento con la formación de 2,3-butanodiol, acetoína y $\mathrm{CO}_{2}$ (gas carbónico) como productos principales. En ambientes con reducción de oxígeno se puede evidenciar crecimiento y fermentación débil en medios que contengan glucosa, por otra parte el comportamiento bioquímico de $B$. brevis no relacionado con su poder biocontrolador esta caracterizado por ser amilasa negativo, caseína negativo, gelatinasa positiva, indol negativo, Voges Proskauer negativo y la mayoría utilizadores de citrato.

Por otra parte, son microorganismos ampliamente distribuidos en el ambiente que suelen encontrarse en el suelo, agua dulce y salada, materia vegetal en descomposición, desiertos y la Antártida. Igualmente B. subtilis se ha aislado en alimentos, incluidas las especias, cacao, legumbres, semillas, y pan. Se encuentra principalmente en suelos agrícolas, raíces de las plantas y en el tracto gastrointestinal de los animales. En diferentes aislamientos se ha evidenciado la presencia de cepas con actividad biocontroladora sobre patógenos de plantas (10), además poseen un alto poder de adaptación a diversos ambientes mediante la formación de endosporas; estructuras resistentes frente a la falta de nutrientes y situaciones adversas. Las esporas poseen la capacidad de diseminarse en el aire, por tanto pueden migrar grandes distancias y ser ubicuas en el medio ambiente hasta encontrar las condiciones óptimas para su crecimiento (10), Figuras 1 y 2 .

\section{Acción biocontroladora: metabolitos} voraces

Se considera mecanismo de biocontrol al uso del conjunto de reacciones metabólicas, bioquímicas, mecánicas y/o físicas que de manera natural se desarrollan articulada o individualmente y desencadenan la inhibición de la expresión de un microorganismo patógeno por parte de otro en un ambiente determinado con el fin de lograr la eliminación parcial o total de este sin emplear agentes químicos que causen efectos adversos.

La acción biocontroladora de Bacillus brevis y Bacillus subtilis esta mediada por la producción de metabolitos antibióticos capaces de actuar sobre microorganismos de diversa etiología; la antibiosis. Los péptidos que produce y que tienen esta acción son variados y representan un grupo no muy heterogéneo entre sí de metabolitos activos que afectan directamente a algunos fitopatógenos como Fusarium oxysporum (10-15). Se ha reportado desde hace más de siete décadas que Bacillus brevis produce gramicidina S (GS). Según Vandamme y Demain (16) en 1976 la gramicidina $S$ (GS) es producto de la expresión de los genes GrsA y GrsB (17), secuencias codificantes de las sintetasas (I y II) con la función de traslocar los aminoácidos en las posiciones especificas dentro del pentapéptido en formación; dos cadenas idénticas de secuencia (DPhe -Lpro -Lval -Lorn -LLeu), el primer aminoácido activado por la sintetasa II y los cuatro restantes por la sintetasa I.

En este mismo estudio, Vandamme y Demain (16) realizaron un análisis comparativo de la relación entre el crecimiento de la población bacteriana y la capacidad productora del antimicrobiano in vitro con la cepa ATCC 9999 en medios enriquecidos con componentes orgánicos; disacáridos, polisacáridos, hexosas, pentosas y ácidos orgánicos. El glicerol y la D- fructosa mostraron ser inhibidores de la producción de GS, de igual forma se observó que aminoácidos especialmente la fenilalanina, así como otros sustratos permiten fases aceleradas en la cinética de crecimiento y producción del antimicrobiano (16).

La función biológica de la GS es la inhibición selectiva de la RNA polimerasa. Sarkar, Langley y Paulus (18) demostraron que a través del aislamiento y purificación de la polimerasa de la cepa de $B$. 

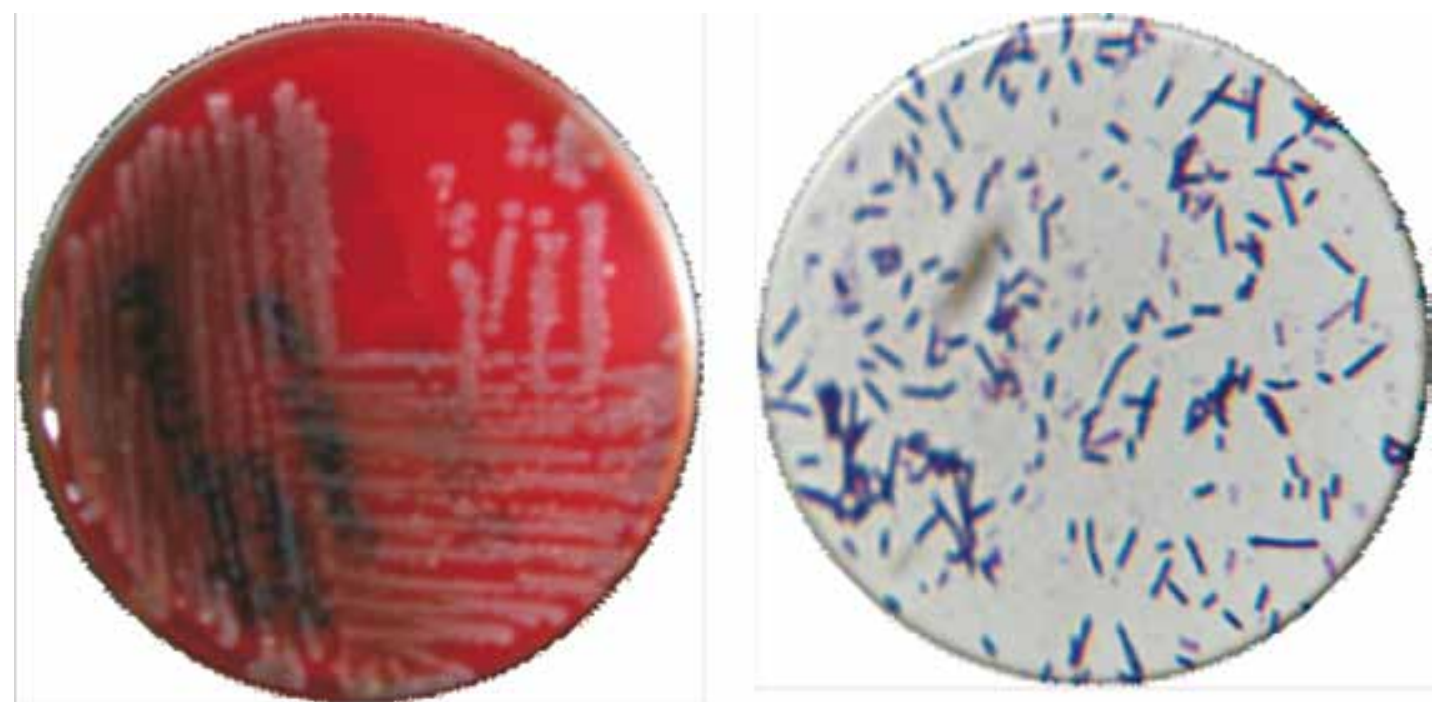

Figura 1. B. subtilis (izquierda); crecimiento de colonias características; grandes, uniformes, de aspecto blanquecino, $\beta$ - hemolíticas. Cultivo en agar sangre a las 24 horas de incubación y B. subtilis (Derecha); Bacilos gram positivos no esporulados. Coloración de Gram. 100X
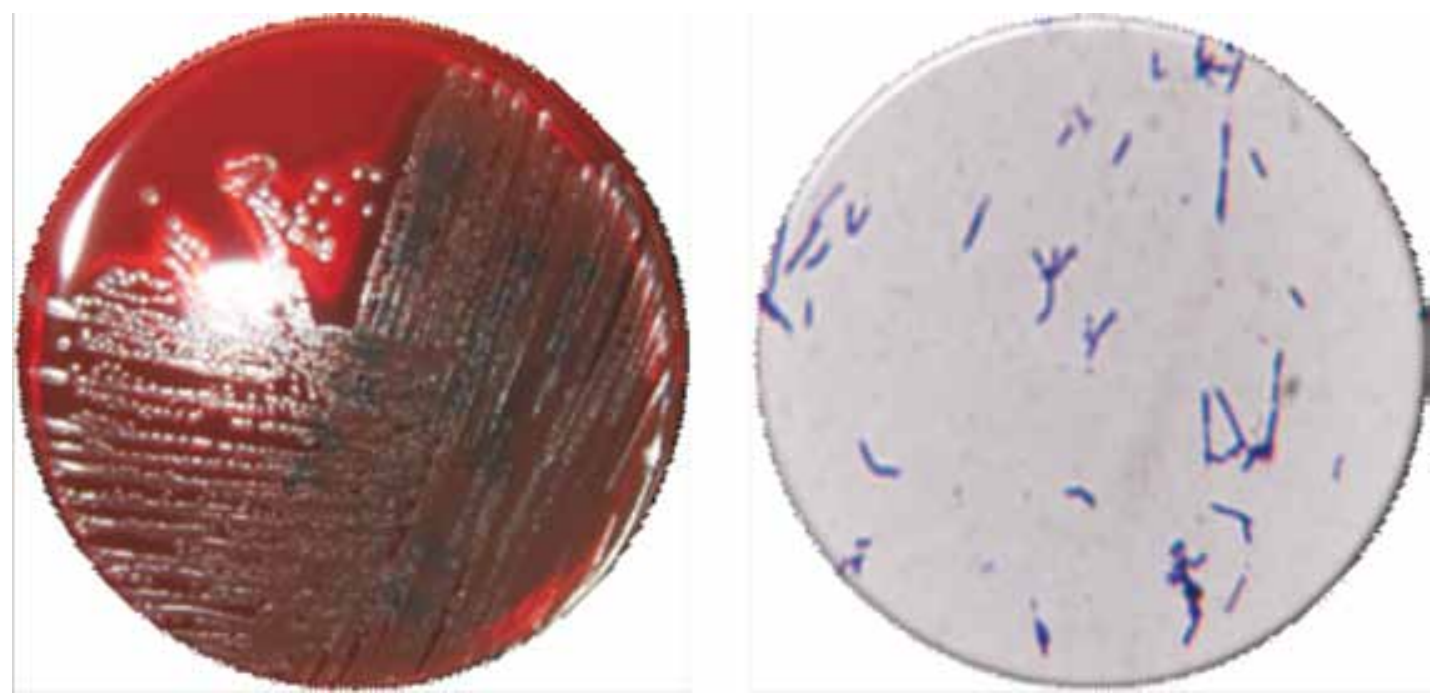

Figura 2. B. brevis (izquierda). Crecimiento de colonias características; grandes, uniformes, de aspecto blanquecino, $\beta$ - hemolíticas. Cultivo en Agar Sangre a las 24 horas de incubación y B. brevis (Derecha); Bacilos gram positivos no esporulados. Coloración de Gram. 100X

brevis ATCC 8185 adicionada a cepas de E. coli y bacteriófago T7, se logró la inhibición de la síntesis de RNA en estos dos microorganismos por acción directa sobre la RNA polimerasa y por tanto la inhibición de su expresión, contrario a lo que ocurre cuando se adiciona la polimerasa purificada al DNA del mismo género bacteriano (18). Hecho que llevo a comprender su mecanismo de acción estableciendo que está mediado por el incremento en la permeabilidad de la membrana de la bacteria blanco, para permitir el ingreso de cationes inorgánicos que se unen a la membrana celular y estructuran una red de canales que atraviesan la membrana lipídica. Por otro parte, dos moléculas de gramicidina $\mathrm{A}$ forman un canal que se fija a la membrana celular y conforman un puente de hidrogeno en sus extremos terminales en forma de un dímero que se extiende a toda la membrana de esta forma los iones se pueden difundir a través del canal acuoso formado. La asociación y disociación de los monómeros del antimicrobiano controla la velocidad del flujo iónico (18).

Basados en estos resultados la producción de GS in vitro depende en gran medida de los sustratos nutricionales de los que disponga $B$. brevis. Sin 
embargo in vivo su producción se efectúa bajo condiciones normales por la expresión genética de locus asociados, sujetos a un aumento progresivo de su expresión por el estimulo directo de un fitopatógeno o incluso otro microorganismo que no pertenece a su género. Su importancia en el control de otros agentes biológicos se extiende no solo a bacterias Gram negativas sino también a parásitos tisulares como el Plasmodium mediado por la producción de tirocidina, complejo de péptidos antibióticos muy relacionados y reportados desde 1944, que tienen actividad antimalárica en glóbulos rojos de pollo infectados con Plasmodium gallinaceum (20).

Por otro lado, Bacillus subtilis posee mecanismos similares respecto a Bacillus brevis, ha demostrado eficiencia in vitro en el control de más de 23 tipos de patógenos de plantas, la competencia frente a otros microorganismos patógenos, se da mediante la secreción de diversas sustancias que se producen cuando la bacteria recibe los nutrientes presentes en la superficie de las raíces de las plantas que induce la elaboración de metabolitos secundarios con capacidad de suprimir el crecimiento de hongos, oomicetos y bacterias fitopatógenas (10). En general la actividad biocontroladora la ejerce mediante la producción de lipopéptidos cíclicos antibióticos (CLPS) entre los cuales se destacan el surfactin y el Iturin A (10); sustancias que han demostrado amplio espectro de acción sobre patógenos de plantas, en los que se encuentran especies de Fusarium, Pythium, Phytophthora, Rhizoctonia, Sclerotinia, Septoria y Verticillium.

El efecto biocontrolador, resulta como producto de diversos mecanismos, entre ellos la antibiosis que ejerce Bacillus subtilis con la producción de péptidos, lipopéptidos y fosfolípidos que se utilizan como agentes terapéuticos contra bacterias patógenas y hongos (10). Estos compuestos tienen origen peptídico y pueden ser sintetizados o no en el ribosoma. Entre las sustancias que no son sintetizados en el ribosoma se destacan los lipopéptidos como el surfactin, Iturin, y fengycin. El ribosoma realiza la síntesis durante el crecimiento activo de las bacterias, a diferencia de los que no son sintetizados por el ribosoma que se producen después del crecimiento bacteriano (10).

El grupo Iturin comprende una serie de compuestos clasificados de A-E. El Iturin A es un antibiótico lipopéptidico cíclico que contiene siete residuos de L-o D- $\alpha$-aminoácidos y un residuo de un ácido graso $\beta$-amino (21-23). En este grupo de antibióticos se han reportado ocho diferentes tipos de Iturin A con base en sus cadenas laterales alquilo. Se ha descrito que la actividad antifúngica está estrechamente relacionada con la longitud de la cadena lateral alquílica $(24,25,26)$. Teniendo en cuenta este marco referencial Iwase et.al analizaron la relación entre la actividad antifungica del Iturin A y la longitud de la cadena lateral alquílica, encontrando que en efecto las isoformas con mayor longitud en ésta, poseían un amplio efecto antagónico antifúngico, por lo que establecieron las características de cultivo ideales para favorecer la producción de isoformas con estas propiedades (26).

Kinsella et al identificaron que la producción de los lipopéptidos antibióticos y la actividad de control biológico están directamente relacionados con la capacidad de Bacillus subtilis de formar biofilms estables en las raíces de las plantas (27). Sin embargo, este microorganismo posee otro tipo de características que probablemente determinan su eficacia relacionada con el amplio espectro de acción y la resistencia a la hidrólisis por peptidasas y proteasas, de igual forma presenta resistencia a altas temperaturas y a una amplia gama de $\mathrm{pH}$ (28). Todos los genes involucrados en la síntesis de antibióticos de Bacillus subtilis ascienden a $350 \mathrm{~kb}$. Es importante resaltar que un promedio entre 4-5\% del genoma de este microorganismo se encarga de la producción de antibióticos (28).

La actividad antimicrobiana de los lipopéptidos se da por la interacción con la membrana de las células diana, en la cual se modifica la permeabilidad y la composición de los lípidos de la membrana con la formación de pequeñas vesículas y la agregación de partículas intramembranosas, de esta forma inhiben el crecimiento del micelio y el desarrollo de los hongos $(25,29)$. Las variaciones en la longitud y ramificación de las cadenas de ácidos grasos, así como las sustituciones de aminoácidos permiten la diferenciación de los lipopéptidos identificados hasta ahora en Bacillus subtilis los cuales se dividen en tres grupos: la familia de surfactin, Iturin y fengycin (28). Estos antibióticos presentan 
estructuras de tipo terciario y cuaternario (28).

El lipopéptido antibiótico fengicina es sintetizado por una enzima compleja formada por cinco sintetasas, que incluyen: FenA, FenB, FenC, FenD y FenE; codificadas por el operon fengycin sintetasa (FEN). Este operon está formado por una secuencia de ácidos grasos L-Glu-D-Orn-L-Tyr-D-allo-Thr-LGlu-Ala-D (D-Val)-L-Pro-L-Glu-Tyr-D-L-Ile, con un enlace de conexión L-lactona Tyr e Ile $(30,31)$.

La estructura del fengycin se compone de un ácido graso b-hidroxilo conectado a la $\mathrm{N}$-terminal de un péptido que contiene 10 aminoácidos incluyendo cuatro residuos de ácido D-aminoácidos y el aminoácido L-ornitina. En la mitad está ubicado un residuo C-terminal que está relacionado con los residuos de tirosina en la posición 3. Este lipopéptido posee una actividad fungicida contra los hongos filamentosos y tiene actividad hemolítica 40 veces menor que la del surfactin $(31,32,33)$.

Es así como Iturin A, Fengycin y Gramicidina S se constituyen como metabolitos de alta actividad contra microorganismos en diversas etiologías y representan un importante eslabón en el biocontrol de plagas (34-37).

\section{Los BCAs: ila panacea?}

Los avances conseguidos en la actualidad en materia de mejoramiento y viabilidad de los suelos indicados dentro del fortalecimiento de la práctica agrícola incluye el aumento del uso de agentes de control biológico (BCAs), como estrategia fundamental para el control de fitopatógenos y la reducción de los efectos adversos que causan los agroquímicos. Estos microorganismos marcan la pauta para dimensionar. Torres y Capote (38) han documentado la problemática ambiental actual, en la cual los altos niveles de contaminación producto del uso extensivo de compuestos químicos se ha convertido desde hace más de siete décadas en una constante generadora de partículas contaminantes que se dispersan sobre los seres humanos que los manipulan, la flora y fauna en mayor proporción incluso, que la que afecta directamente al blanco de acción esperado. Esta problemática en países industrializados ha llevado a regular y anular su uso. Sin embargo en países tropicales en vía de desarrollo como Colombia, la

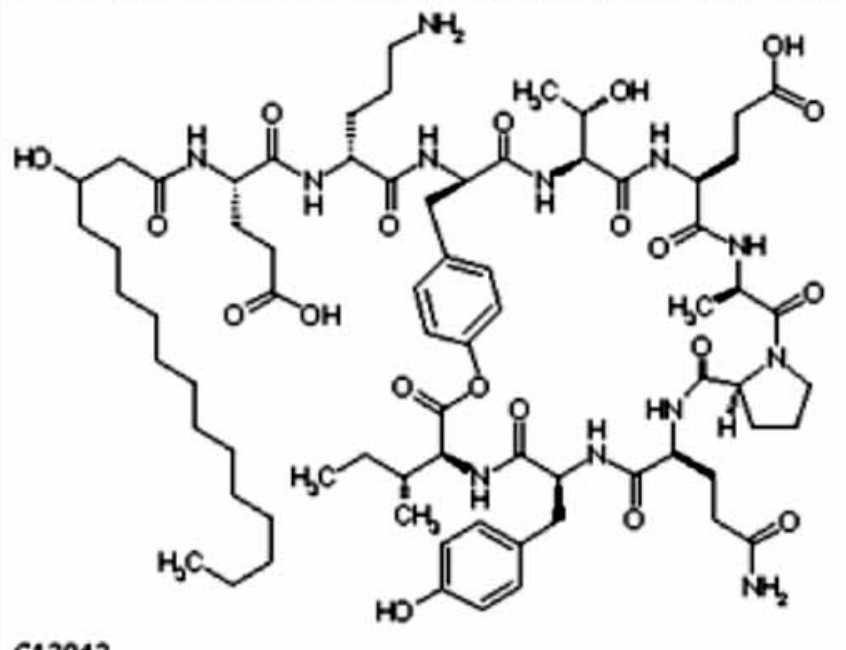

Figura 3. Estructura química del fengicina (The American Chemical Society 2010).

situación es diferente a pesar de la implementación del marco legal que regula el registro, control y venta de compuestos químicos y pesticidas a través de la Ley 822 de 2003, por ello el espectro de acción de los BCAs es un campo importante de exploración y una necesidad latente.

Nagórska, Bikowski y Obuchowski (39) refieren que desde finales de 1900 se han venido empleando microorganismos nativos del suelo, como protección para las plantas afectadas por hongos, con el fin de disminuir los efectos adversos antes mencionados. De acuerdo con esta propuesta se ha implementado el uso de agentes biocontroladores de fitopatógenos como Bacillus subtilis y Bacillus brevis, por la demostrada capacidad de producir una amplia gama de sustancias biológicamente activas.

\section{Fusarium sp: el antagonista}

Steinkellner, Mammerler y Vierheilig (40) describen a Fusarium oxysporum como un hongo cosmopolita que se encuentra en la rizósfera, coloniza distintas especies de plantas, sobre las cuales actúa como patógeno causante de marchitez vascular, pudrición de raíz, pudrición de la corona y caída de plántulas. El proceso fitopatológico que tiene lugar en las plantas según Leong, Latiffah y Baharuddin (41) inicia cuando Fusarium oxysporum infecta la planta desencadenando distintos mecanismos de ataque por el sistema inmune de la planta como la secreción de 
una sustancia gelatinosa que permite la formación de tilosa en los vasos y finalmente colonizar el sistema vascular bloqueando el flujo normal del agua hacia la parte superior de la planta con la consecuencia de amarillamiento, marchitez y muerte.

\section{Fusarium spp Vs. Bacillus sp}

Las conclusiones de previas investigaciones como la de Vanegas (2006) et al. señalan que: "Existen cepas bacterianas silvestres del género Bacillus que son capaces de inhibir el crecimiento in vitro de cepas patógenas de F. solani y F. oxysporum" (42) argumento que demuestra que cepas silvestres del género Bacillus sp aislados de la rizósfera de plantas son capaces de generar un efecto antagónico en el crecimiento y desarrollo de este patógeno, además de no presentar efectos adversos en la viabilidad de las plantas del estudio. Este efecto, se realiza a través de mecanismos naturales entre los que se destaca de manera importante la producción de péptidos antimicrobianos como la Gramicidina S, el Iturin A y el Fengycin (43, 44, 45).

Los estudios reportados por Haggag (46) en cultivos de fresa afectados por Botrytis, mencionan la purificación y extracción del péptido de la GS a partir de cultivo de Bacillus brevis por cromatografía en sílice. Los cultivos de este fitopatógeno aislado de las plantas afectadas sometidos a tratamiento con la gramicidina $S$ mostraron la inhibición del crecimiento en todos los casos y en el escaneo con microscopía electrónica se evidencio la absorción de grandes cantidades de GS en suspensión por parte de las plantas, lo que concluye que el tratamiento con B. brevis sobre cultivos afectados generaron un alto impacto contra el fitopatógeno a concentraciones mínimas y redujeron significativamente la incidencia de la enfermedad. Este efecto antagónico posee un amplio rango de acción que no esa limitado solo al género Botrytis, soporte científico que demuestra el gran potencial biocontrolador de B. brevis para inhibir el crecimiento de Fusarium spp.

Uno de los estudios más significativos en términos de reproducibilidad de los resultados que se realizo en nuestro país por el grupo de investigación CEPARIUM de la Universidad Colegio Mayor de Cundinamarca por Bautista, González y Guevara en 2010 y en la cual se estableció que "Cuatro aislamientos de Bacillus spp., presentaron actividad antagónica in vitro frente a Fusarium sp., con un porcentaje de inhibición mayor al 45\%, mediada por la secreción de sustancias antifúngicas propias del género" y que "los cuatro aislamientos de Bacillus spp., presentaron efecto biocontrolador bajo condiciones de invernadero sobre Fusarium sp., en romero, al disminuir la severidad de la marchitez vascular en las plántulas por debajo del 50\%" (47), resultados que en la actualidad ya permiten adelantar las técnicas moleculares para establecer los mecanismos de acción específicos utilizados por el género bacteriano.

\section{Otros mecanismos antagónicos}

A pesar de que la antibiosis sea el mecanismo antagónico más utilizado por los microorganismos biocontroladores para inhibir al fitopatógeno, no es el único, de hecho los antagonistas no tienen un solo modo de acción y esto les brinda una característica importante para ser usados como agentes de control biológico (48). Por esta razón, se han descrito varios mecanismos antagónicos empleados por microorganismos que controlan el desarrollo de patógenos; "Algunos de estos son competencia por espacio o por nutrimentos, interacciones directas con el patógeno (micoparasitismo y lisis enzimática) e inducción de resistencia” (48). En general dentro de los principales mecanismos de acción se encuentran:

Competencia: Este mecanismo consiste en el uso de un requerimiento en común por dos especies, con la diferencia que uno de ellos hace mayor uso de este que el otro, limitando la cantidad de requerimiento disponible y generando un evento desigual (48).

Competencia por nutrimentos: La competencia más común es por nutrimentos esenciales para el desarrollo de las funciones microbianas vitales; reproducción, nutrición, respiración y/o metabolismo, de esta manera se delimita la colonización de otras especies patógenas (48).

Competencia por espacio: El desarrollo de un microorganismo sobre determinada área, inhibe de cierto modo la invasión de otros (48).

Interacción directa con el patógeno: Se destacan dos tipos de interacción directa entre los antagonistas y los patógenos el parasitismo y la predación (49), la primera se considera por varios autores como una 
simbiosis antagónica entre organismos; la capacidad que posee uno de ellos denominado antagonista de alimentarse de otro microorganismo, que en el contexto del biocontrol es un agente patógeno. Este mecanismo esta mediado básicamente por la lisis enzimática, la cual permite la degradación de las estructuras fúngicas parasitadas para su utilización como fuente de factores nutricionales ya que son exoproteasas principalmente que hidrolizan los enlaces peptídicos hasta dipéptidos, tripéptidos y aminoácidos libres que usa el antagonista para su alimentación, dentro de las más importantes las quitinasas, celulasas, proteasas y la $\beta-1,3$ glucanasa. Existen por tanto dos tipos de lisis: la endolisis y la exolisis, la primera consiste en la destrucción del citoplasma de una célula por sus propias enzimas y la segunda se produce debido a enzimas o toxinas de otros organismos (50).

Por otro lado la predación; consiste en la utilización de materia orgánica como fuente nutricional por parte del antagonista, de la que puede hacer parte el patógeno, lo cual es muy ocasional. Como ejemplo, en estudios anteriores se ha descrito la presencia de amebas en suelo que ejercen efectos supresores de enfermedad y lo protegen de la proliferación de microorganismos no deseados al alimentarse de las hifas de hongos patógenos (51).

Protección cruzada e Inducción de resistencia: Los seres vivos incluidas las plantas han desarrollado mecanismos de defensa contra los patógenos invasores en el transcurso del tiempo y el proceso evolutivo. Por tal motivo las plantas presentan mecanismos bioquímicos, físicos y estructurales de resistencia los cuales están muy relacionados con la capacidad de éstas para inhibir el desarrollo de una enfermedad por fitopatógenos con los que no han estado en contacto mediadas por los mecanismos preexistentes desencadenados por otro patógeno similar (51) conocido como el fenómeno de la protección cruzada.

La resistencia inducida es un tipo particular de este fenómeno, en la cual los mecanismos de defensa reconocen y responden a amenazas similares y están preparados antes de la amenaza real dada por la llegada del patógeno, esta protección cruzada puede por tanto estar dada por la inducción de resistencia u otros mecanismos antagónicos (51).

\section{Discusión}

La síntesis y ensamblaje de polipéptidos antimicrobianos como las iturinas, fengicinas y gramicidinas requieren de la expresión de varios genes codificados en el genoma bacteriano que intervienen en su formación, principalmente de tipo sintetasas. La presencia de estos genes asociados en cepas de Bacillus $s p p$. de origen nativo, preferiblemente, es un factor determinante para establecer si la antibiosis es el mecanismo de acción que induce muerte al fitopatógeno, teniendo en cuenta que es el utilizado por la mayoría de las cepas. Sin embargo, esta identificación debe ser complementada con análisis de expresión génica; cuantificación de ARNm o aislamiento y purificación del péptido por cromatografía o Western Blot que podrían ser efectivas para establecer si los genes son productores de los polipéptidos en mención.

Por tanto, las técnicas moleculares al servicio de la agricultura ofrecen un amplio panorama para el conocimiento más profundo de todos los mecanismos que intervienen en las relaciones antagónicas. Según Devendra y col.(52) la resistencia sistémica inducida (RSI) por especies bacterianas asociadas con plantas por varias cepas de especies de B. amyloliquefaciens, $B$. subtilis, B. pasteurii, B. cereus, B. pumilus, $B$. mycoides y $B$. sphaericus reducen significativamente la incidencia o la gravedad de varias enfermedades en diversidad de cultivos, lo que debe generar el uso progresivo y más eficiente de estas a través del uso de técnicas moleculares con un enfoque estratégico de control biológico (52).

\section{Conclusiones}

Bacillus spp y sus especies más representativas $B$. brevis y $B$. subtilis, corresponden a dos agentes bacterianos con amplias y excepcionales acciones antagónicas contra microorganismos de diversas etiologías, lo que sugiere la explotación de su metabolismo y perfil genético en el biocontrol de plagas y agentes fitopatógenos.

Fusarium sp es un agente fitopatógeno causante de marchitez vascular y pudrición de raíz, procesos de enfermedad que tienen lugar en plantas aromáticas y ornamentales y que conllevan a disminuir 
su producción y la calidad para exportación y comercialización.

Los péptidos antimicrobianos Iturin A, Fengycin y Gramicidina $S$ son el producto de la interacción de varios locus genéticos existentes en el genoma bacteriano de estas especies $(53,54)$ y su producción está dada no solo por las características intrínsecas que poseen por naturaleza si no en la misma medida por el estimulo directo de otro microorganismo como es que posea una relación de antagonismo a través de la activación del sistema de comunicación y señalización bacteriano $(55,56)$.

El uso a mediano plazo de cepas que demuestren efectos antagónicos en programas de biocontrol es una de las herramientas más próximas y efectivas para contribuir en la disminución de las actuales problemáticas fitosanitarias.

\section{Referencias}

1. Thasana N, Prapagdee B, Rangkadilok N, Sallabhan R, Aye SL, Ruchirawat $S$, Loprasert S. Bacillus subtilis SSE4 produces subtulene A, a new lipopeptide antibiotic possessing an unusual $\mathrm{C} 15$ unsaturated beta-amino acid. FEBS Lett. [En línea]. Julio 16 de 2010 [Revisado el 10 de Enero de 2011] 584(14):3209-14; Disponible en: http://www.ncbi.nlm.nih. gov/pubmed/20541548

2. Kim PI, Ryu J, Kim YH, Chi YT. Production of biosurfactant lipopeptides Iturin A, fengycin and surfactin A from Bacillus subtilis CMB32 for control of Colletotrichum gloeosporioides. J Microbiol Biotechnol. [En línea]. Junio de 2010 [Revisado el 10 de Enero de 2011] 20(1):13845.; Disponible en: http://www.ncbi.nlm.nih.gov/pubmed/20134245

3. Arguelles-Arias A, Ongena M, Halimi B, Lara Y, Brans A, Joris B, Fickers P. Bacillus amyloliquefaciens $\mathrm{GA} 1$ as a source of potent antibiotics and other secondary metabolites for biocontrol of plant pathogens. [En línea]. Noviembre de 2009 [Revisado el 10 de Enero de 2011] 8:63.; Disponible en: http://www.ncbi.nlm.nih.gov/pubmed/19941639

4. Grover M, Nain L, Singh SB, Saxena AK. Molecular and biochemical approaches for characterization of antifungal trait of a potent biocontrol agent Bacillus subtilis RP24. Curr Microbiol. [En línea]. Febrero de 2010 [Revisado el 10 de Enero de 2011] 60(2):99-106.; Disponible en: http:// www.ncbi.nlm.nih.gov/pubmed/19777301

5. Shakerifard P, Gancel F, Jacques P, Faille C. Effect of different Bacillus subtilis lipopeptides on surface hydrophobicity and adhesion of Bacillus cereus 98/4 spores to stainless steel and Teflon. Biofouling. [En línea]. 2009 [Revisado el 10 de Enero de 2011] 25(6):533-41..; Disponible en: http://www.ncbi.nlm.nih.gov/pubmed/19431000

6. Jourdan E, Henry G, Duby F, Dommes J, Barthélemy JP, Thonart P, Ongena $\mathrm{M}$. Insights into the defense-related events occurring in plant cells following perception of surfactin-type lipopeptide from Bacillus subtilis. Mol Plant Microbe Interact. [En línea]. Abril de 2009 [Revisado el 09 de Enero de 2011] 22(4):456-68; Disponible en: http://www.ncbi.nlm. nih.gov/pubmed/19271960

7. Czaczyk K, Białas W, Myszka K. Cell surface hydrophobicity of Bacillus $s p p$. as a function of nutrient supply and lipopeptides biosynthesis and its role in adhesion. Pol J Microbiol. [En línea]. 2008 [Revisado el 09 de Enero de 2011] 57(4):313-9; Disponible en: http://www.ncbi.nlm.nih. gov/pubmed/19275045
8. Hsieh FC, Lin TC, Meng M, Kao SS. Comparing methods for identifying Bacillus strains capable of producing the antifungal lipopeptide iturin A. Curr Microbiol. [En línea]. 2008 [Revisado el 09 de Enero de 2011] 56(1):1-5; Disponible en: http://www.ncbi.nlm.nih.gov/ pubmed/18027023

9. Edwards M, Bacterial physiology: a molecular approach. Ed. El-SharoudSpringer- Verlag Berlin Hiedelberg; 2008

10. Raghavendra J, Brian B. Identification and Characterization of Novel Genetic Markers Associated with Biological Control Activities in Bacillus subtilis. E-Xtra [en línea], 2005 [Revisado el 14 de Agosto de 2010]; 96 (2). Disponible en: http://apsjournals.apsnet.org/doi/pdf/10.1094/ PHYTO-96-0145

11. Kamysz E, Mickiewicz B, Kamysz W, Bielińska S, Rodziewicz-Motowidło S, Ciarkowski J. Synthesis, biological activity and solution structure of new analogues of the antimicrobial Gramicidin S. J Pept Sci [en línea]. Octubre 25 de 2010 [Revisado el 10 de Enero de 2011]; Disponible en: http://www.ncbi.nlm.nih.gov/pubmed/20976832

12. Vogt TC, Schinzel S, Bechinger B. Biosynthesis of isotopically labeled gramicidins and tyrocidins by Bacillus brevis. J Biomol NMR. [en línea]. Mayo 26 de 2003 [Revisado el 10 de Enero de 2011] 26(1):1-11; Disponible en: http://www.ncbi.nlm.nih.gov/pubmed/12766398

13. Edwards SG, Seddon B.Selective medium based on tyrosine metabolism for the isolation and enumeration of Brevibacillus brevis (Bacillus brevis). Lett Appl Microbiol. [en línea]. Noviembre de 2000 [Revisado el 10 de Enero de 2011] 31(5):395-9; Disponible en: http://www.ncbi.nlm.nih. gov/pubmed/11069644

14. Berditsch M, Afonin S, Ulrich AS. The ability of Aneurinibacillus migulanus (Bacillus brevis) to produce the antibiotic gramicidin $S$ is correlated with phenotype variation. Appl Environ Microbiol [en línea]. Octubre de 2007 [Revisado el 10 de Enero de 2011] 73(20):6620-8; Disponible en: http://www.ncbi.nlm.nih.gov/pubmed/17720841

15. Bohg A, Ristow H. DNA-supercoiling is affected in vitro by the peptide antibiotics tyrocidine and gramicidin. Eur J Biochem [en línea].Noviembre 31986 [Revisado el 10 de Enero de 2011] 160(3):587-91; Disponible en: http://www.ncbi.nlm.nih.gov/pubmed/2430800

16. Vandamme E., Demain A. Nutrition of bacillus brevis ATCC 9999, the producer of Gramicidin S. Antimicrobial agents and chemotherapy [en línea]. 1976. [Revisado el 9 de Marzo de 2010]; Vol. 10, No. 2. Disponible en:http://www.ncbi.nlm.nih.gov/pmc/articles/PMC429733/

17. Kratzschmar J., Krause M., Marahiel M. Gramicidin S biosynthesis operon containing the structural genes GRSA and GRSB has an open reading frame encoding a protein homologous to fatty acid thioesterases. Journal of Bacteriology [en línea]. 1989. [Revisado el 9 de Mayo de 2010]; Vol. 171, No. 10. Disponible en: http://www.ncbi.nlm.nih.gov/pubmed/2477357

18. Sarkar N, Langley D, Paulus H, Biological function of gramicidin: Selective inhibition of RNA polymerase. Proceedings of the National Academy of science of the United States of America. Revista Pub Med Central [en línea], 1977 [Revisado el 14 de Agosto de 2010]; 74 (4) Disponible en: http://www.ncbi.nlm.nih.gov/pmc/articles/PMC430807/

19. Wiley J y sons Inc, Textbook of biochemestry with clinic correlations. Fifth edition. Ed. Reverte Barcelona- España; 2004

20. Rautenbach M, Maré V, Stander M, Hoppe H, Inhibition of malaria parasite blood stages by tyrocidines, membrane-active cyclic peptide antibiotics from Bacillus brevis. Biochimica et Biophysica Acta (BBA) [en línea] 2007 [Revisado el 14 de Junio de 2010] 1768 (6): 1488-1497 Disponible en: http://www.sciencedirect.com/science? ob=ArticleURL

21. Li Q, Meng X, Wu X, Lin W, et al. Purification of Two Antimicrobial Substances Produced by Bacillus subtilis Strain B11 and Their Properties. Agricultural Sciences in China [en línea], 2006 [Revisado Mayo 21 de 2010]; 5(5). Disponible en: http://www.sciencedirect.com/ science? ob=ArticleURL\& udi=B82XG-4K30SB2-6\& user $=10 \&$ coverDate $=05 \% 2 \mathrm{~F} 31 \% 2 \mathrm{~F} 2006 \&$ rdoc $=1 \& \mathrm{fmt}=$ high $\&$ orig $=$ search $\&$ sort $=\mathrm{d} \&$ docanchor $=\&$ view $=c \&$ search $S t r I d=1440896467 \&$ rerunOrigin $=$ google $\&$ acct $=\mathrm{C} 000050221 \&$ version $=1 \&$ urlVersion $=0 \&$ userid $=10 \& m d 5=b 2 a 8 a 48 a 706 c 552 b 1 a e 78 c 162391 \mathrm{a} 4 \mathrm{~d} 7$ 
22. Mizumoto S, Hirai M, Shoda M. Production of lipopeptide antibiotic Iturin A using soybean curd residue cultivated with Bacillus subtilis in solid-state fermentation. Appl Microbiol Biotechnol [En línea], 2006 [Revisado el 9 de Mayo de 2010]; 72: 869-875. Disponible en: http:// www.ncbi.nlm.nih.gov/pubmed/17476498

23. Romero D, de Vicente A, Rakotoaly RH, Dufour SE, Veening JW, Arrebola E, Cazorla FM, Kuipers OP, Paquot M, Pérez-García A.The iturin and fengycin families of lipopeptides are key factors in antagonism of Bacillus subtilis toward Podosphaera fusca. Mol Plant Microbe Interact. [En línea], 2007 [Revisado el 13 de Enero 2011]; 20(4):430-40. Disponible en: http://www.ncbi.nlm.nih.gov/pubmed/17427813

24. Rahman MS, Ano T, Shoda M. Biofilm fermentation of iturin A by a recombinant strain of Bacillus subtilis 168. J Biotechnol [En línea], 2007 [Revisado el 13 de Enero 2011]; 127(3):503-7. Disponible en: http:// www.ncbi.nlm.nih.gov/pubmed/16942812

25. Aranda FJ, Teruel JA, Ortiz A. Further aspects on the hemolytic activity of the antibiotic lipopeptide iturin A. Biochim Biophys Acta [En línea], 2005 [Revisado el 13 de Enero 2011]; 1713(1):51-6. Disponible en: http://www.ncbi.nlm.nih.gov/pubmed/15949788

26. Iwase $\mathrm{N}$, Rahman M, Ano T. Production of iturin A homologues under different culture conditions. Journal of Environmental Sciences Supplement [en linea], 2009 [Revisado 15 de Julio de 2010]; S28-S32. Disponible en: http://www.sciencedirect.com/science?_ob=ArticleURL\&_udi=B8CX44W45TP3-8\& user $=10 \&$ coverDate $=12 \% 2 \mathrm{~F} 31 \% 2 \mathrm{~F} 2009 \&$ rdoc $=1 \&$ fmt $=$ high $\& \_$orig $=$search $\& \_$sort $=\mathrm{d} \& \_$docanchor $=\& v i e w=c \& \_$searchStrId $=1440899794 \&$ rerunOrigin $=$ google $\&$ acct $=C 000050221 \&$ ver-

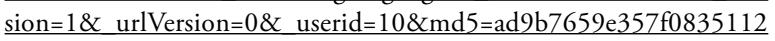
$\underline{831 \mathrm{~b} 75316 \mathrm{~d} 2 \mathrm{f}}$

27. Kinsella K, Schulthess C, Morris T, et al. Rapid quantification of Bacillus subtilis antibiotics in the rhizosphere. Soil Biology \& Biochemistry [en linea], 2009 [Revisado 30 de Junio de 2010]; 41. Disponible en: http://www.sciencedirect.com/science?_ob=ArticleURL\&_udi=B6TC74V7658J-1\&_user $=10 \& \_$coverDate $=02 \% 2 \mathrm{~F} 28 \% 2 \mathrm{~F} 2009 \&$ \& rdoc $=1 \&$ _ fmt=high\&_orig=search\&_sort=d\&_docanchor $=\& v i e w=c \&$ searchStrId $=1440887722 \& \_r e r u n O r i g i n=g o o g l e \& \_a c c t=C 000050221 \& \_v e r-$ sion $=1 \&$ urlVersion $=0 \&$ userid $=10 \& \mathrm{md} 5=\mathrm{c} 842 \mathrm{e} 668 \mathrm{ff} 6 \mathrm{~b} 73 \mathrm{a} 2 \mathrm{fc} 9 \mathrm{~b} 70$ $721 \mathrm{da} 0 \mathrm{ca} 14$

28. Nagórska K, Bikowski M, Obuchowski M. Multicellular behaviour and production of a wide variety of toxic substances support usage of Bacillus subtilis as a powerful biocontrol agent. Acta Biochemical Polonica [en línea]. 2007 [Revisado el 7 de Mayo de 2010]; 54 No. 3: 495-508. Disponible en: http://www.actabp.pl/pdf/3_2007/495s.pdf

29. Gong M, Wang JD, Zhang J, et al. Study of the Antifungal Ability of Bacillus subtilis Strain PY-1 in Vitroand Identification of its Antifungal Substance (Iturin A). Acta Biochimica et Biophysica Sinica [en línea], 2006 [Revisado el 14 de Mayo de 2010]; 38(4): 233-240. Disponible en: http://abbs.oxfordjournals.org/cgi/content/abstract/38/4/233

30. Deleu M,Paquot M,Nylander T. Effect of Fengycin, a Lipopeptide Produced by Bacillus subtilis, on Model Biomembranes. Biophysical Journal [en línea], 2008 [Revisado el 1 de Septiembre de 2010]; 94: 2667-2679. Disponible en: http://www.sciencedirect.com/science? ob=MImg\& imagekey=B94RW $-4 T X 32 \mathrm{HK}-\mathrm{V}-1 \& \_$cdi $=56421 \&$ _user $=10 \&$ _ pii $=$ S0006349508705198\& coverDate $=04 \% 2 \mathrm{~F} 30 \% 2 \mathrm{~F} 2008 \&$ sk $=\% 2$ 3TOC $\% 2356421 \% 232008 \% 23999059992 \% 23701862 \% 23$ FLA $\% 23$ display\%23Volume 94,_Issue_7, Pages_L45-L59, 2443-2925_(April 2008)\%23tagged $\% 23$ Volume $\% 23$ first $\% 3$ D $94 \% 23$ Issue $\% 23$ first $\% 3 \mathrm{D}$ 7\%23date\%23(April_2008)\%23\&view=c\&_gw=y\&wchp=dGLbVzz-zS kzS\&md5=e2d2437a82372473d1b4199b73055133\&ie=/sdarticle.pdf

31. Ju Ke W, Tung Liu S. Regulation of the Transcription of the Fengycin Synthetase Operon. [en línea] [Revisado el 5 de Septiembre de 2010]. Disponible en: http://memo.cgu.edu.tw/research/edu/02ke92.pdf

32. Wei YH, Wang LC, Chen WC, Chen SY. Production and Characterization of Fengycin by Indigenous Bacillus subtilis F29-3 Originating from a Potato Farm. Int J Mol Sci. [En línea], Noviembre 12 de 2010 [Revisado el 13 de Enero 2011] 11(11):4526-38; Disponible en: http://www.ncbi. nlm.nih.gov/pubmed/21151454
33. Bie X, Lu Z, Lu F. Identification of fengycin homologues from Bacillus subtilis with ESI-MS/CID. J Microbiol Methods. [En línea], Diciembre de 2009 [Revisado el 13 de Enero 2011] 79(3):272-8; Disponible en: http://www.ncbi.nlm.nih.gov/pubmed/19781583

34. Tapi A, Chollet-Imbert M, Scherens B, Jacques P. New approach for the detection of non-ribosomal peptide synthetase genes in Bacillus strains by polymerase chain reaction. Appl Microbiol Biotechnol. [En línea], Febrero de 2010 [Revisado el 13 de Enero 2011] 85(5):1521-31.; Disponible en: http://www.ncbi.nlm.nih.gov/pubmed/19730852

35. Chen H, Wang L, Su CX, Gong GH, Wang P, Yu ZL. Isolation and characterization of lipopeptide antibiotics produced by Bacillus subtilis. Lett Appl Microbiol. [En línea], Septiembre de 2008 [Revisado el 13 de Enero 2011] 47(3):180-6..; Disponible en: http://www.ncbi.nlm.nih. gov/pubmed/19552782

36. Ke WJ, Chang BY, Lin TP, Liu ST. Activation of the promoter of the fengycin synthetase operon by the UP element. J Bacteriol. [En línea], 2009 [Revisado el 13 de Enero 2011] 191(14):4615-23; Disponible en: http://www.ncbi.nlm.nih.gov/pubmed/19447911

37. Lin TP, Chen CL, Fu HC, Wu CY, Lin GH, Huang SH, Chang LK, Liu ST. Functional analysis of fengycin synthetase FenD. Biochim Biophys Acta. [En línea], 2005 [Revisado el 13 de Enero 2011] 1730(2):159-64; Disponible en: http://www.ncbi.nlm.nih.gov/pubmed/16102594

38. Torres D., Capote T. Agroquímicos un problema ambiental global: uso del análisis químico como herramienta para el monitoreo ambiental. Ecosistema, Revista científica y técnica de ecología y medio ambiente [en línea], 2004 [Revisado el 11 de Abril de 2010]; 13(3). Disponible en: http://www.revistaecosistemas.net/articulo.asp?Id=50

39. Nagórska K, Bikowski M, Obuchowski M. Multicellular behaviour and production of a wide variety of toxic substances support usage of Bacillus subtilis as a powerful biocontrol agent. Acta Biochemical Polonica [en línea]. 2007 [Revisado el 7 de Mayo de 2010]; 54 No. 3: 495-508. Disponible en: http://www.actabp.pl/pdf/3_2007/495s.pdf

40. Steinkellner S, Mammerler R, Vierheilig H. Germination of Fusarium oxysporum in root exudates from tomato plants challenged with different Fusarium oxysporum strains. Eur J Plant Pathol [en línea]. 2008 [Revisado el 9 de Mayo de 2010]; 122:395-401. Disponible en: http://www. bashanfoundation.org/horst/horststrains.pdf

41. Leong S, Latiffah Z, Baharuddin S. Molecular Characterization of Fusarium Oxysporum F. Sp. Cubense of Banana. American Journal of Applied Sciences [en línea]. 2009 [Revisado el 9 de Mayo de 2010]; 6 (7): 1301-1307. Disponible en: http://www.scipub.org/fulltext/ajas/ ajas671301-1307.pdf

42. Venegas E, Ciampi L., Collado L., Costa M., Fuentes R., Nissen J., Schobitz R., Schoebitz M. Aislamiento e identificación de bacterias nativas del género bacillus cohn antagonistas de cepas patógenas de fusarium link. en cala. Agro sur. [en línea]. 2005 [Revisado el 15 de Mayo de 2010], vol.33, no.2, p.1-12. Disponible: http://mingaonline.uach.cl/scielo. php?script=sci $\quad$ arttext\&pid=S0304-88022005000200001\&lng $=e s \& n r$ $\underline{m=i s o>\text {. ISSN 0304-8802 }}$

43. Liu J, Liu M, Wang J, Yao JM, Pan RR, Yu ZL. Enhancement of the Gibberella zeae growth inhibitory lipopeptides from a Bacillus subtilis mutant by ion beam implantation. Appl Microbiol Biotechnol. [En línea], 2005 [Revisado el 13 de Enero 2011] 69(2):223-8; Disponible en: http:// www.ncbi.nlm.nih.gov/pubmed/15838674

44. Deleu M, Paquot M, Nylander T. Fengycin interaction with lipid monolayers at the air-aqueous interface-implications for the effect of fengycin on biological membranes. J Colloid Interface Sci. [En línea], 2005 [Revisado el 13 de Enero 2011] 283(2):358-65; Disponible en: http://www.ncbi. nlm.nih.gov/pubmed/15721905

45. Ongena M, Duby F, Jourdan E, Beaudry T, Jadin V, Dommes J, Thonart P. Bacillus subtilis M4 decreases plant susceptibility towards fungal pathogens by increasing host resistance associated with differential gene expression. Appl Microbiol Biotechnol. [En línea], 2005 [Revisado el 13 de Enero 2011] 67(5):692-8; Disponible en: http://www.ncbi.nlm.nih. gov/pubmed/15578181 
46. Haggag W. Isolation of bioactive antibiotic peptides from Bacillus brevis and Bacillus polymyxa against Botrytis grey mould in strawberry. Archives of Phytopathology and Plant Protection. [en línea]. 2008. [Revisado el 9 de Mayo de 2010] 41(7): 477 - 491 Disponible en: http://www. informaworld.com/smpp/content $\sim$ content $=\mathrm{a} 769582525$

47. Bautista, Gonzales, Guevara, Efecto biocontrolador de Bacillus spp., frente a Fusarium sp., bajo condiciones de invernadero en romero (Rosmarinus officinalis L.)

48. Cook R.S Y K.F Baker. The nature and practice of biological control of plant pathogens. The American Phytopathological Society, St.Paul. 1983. $539 \mathrm{pp}$.

49. Lecuona, RE, Microorganismos patógenos empleados en el control microbiano de insectos plaga. Ed. 1996. Argentina; 338 p.

50. Vega L, Fernández O, Microorganismos Antagonistas Para El Control Fitosanitario, Manejo Integrado De Plagas (Costa Rica), [En línea] 2001 [Revisado 6 de Marzo de 2010]; No.62, 96 - 100, Disponible en: http:// web.catie.ac.cr/informacion/rmip/rev62/96-100.pdf

51. Campell R, Biological control of microbial plant pathogens. New York: Cambridge University Press; 1989.

52. Devendra K, Bhavdish J, Interactions of Bacillus spp. and plants - With special reference to induced systemic resistance (ISR), Microbiological Research [En línea], 29 de Septiembre de 2009 [Revisado el 14 de Abril de 2011] 164; 5 493-513. Disponible en: http://www.sciencedirect.com/science?_ob=ArticleURL\&_udi=B7GJ8-4TMHKSY-1\&_ user $=10 \& \_$coverDate $=09 \% 2 \mathrm{~F} 29 \% 2 \mathrm{~F} 2009 \& \_\mathrm{rdoc}=1 \& \_\mathrm{fmt}=\mathrm{high} \&$ orig=gateway $\& \_$origin $=$gateway $\& \_$sort $=\mathrm{d} \& \_$docanchor $=\&$ view $=\mathrm{c} \& \_$ searchStrId $=1718834429 \&$ rerunOrigin $=$ scholar.google \&

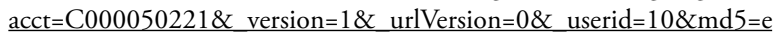
42dceb4b9ef002ad74e3bad97cd807d\&searchtype $=\mathrm{a}$

53. Nam MH, Park MS, Kim HG, Yoo SJ. Biological control of strawberry Fusarium wilt caused by Fusarium oxysporum $f$. sp. fragariae using Bacillus velezensis BS87 and RK1 formulation. J Microbiol Biotechnol. [En línea].2009 [Revisado el 10 de Enero de 2011] 19(5):520-4; Disponible en: http://www.ncbi.nlm.nih.gov/pubmed/19494701

54. Raddadi N, Belaouis A, Tamagnini I, Hansen BM, Hendriksen NB, Boudabous A, Cherif A, Daffonchio D. Characterization of polyvalent and safe Bacillus thuringiensis strains with potential use for biocontrol. J Basic Microbiol. [En línea].2009 [Revisado el 10 de Enero de 2011] 49(3):293303.; Disponible en: http://www.ncbi.nlm.nih.gov/pubmed/19025870

55. Ortega-Morales BO, Ortega-Morales FN, Lara-Reyna J, De la RosaGarcía SC, Martínez-Hernández A, Montero-M J. Antagonism of Bacillus $s p p$. isolated from marine biofilms against terrestrial phytopathogenic fungi. Mar Biotechnol (NY). [En línea].2009 [Revisado el 10 de Enero de 2011] 11(3):375-83.; Disponible en: http://www.ncbi.nlm.nih.gov/ pubmed/18931878

56. Pryor SW, Gibson DM, Hay AG, Gossett JM, Walker LP. Optimization of spore and antifungal lipopeptide production during the solid-state fermentation of Bacillus subtilis. Appl Biochem Biotechnol. [En línea].2007 [Revisado el 10 de Enero de 2011] 143(1):63-79.Disponible en: http:// www.ncbi.nlm.nih.gov/pubmed/18025597
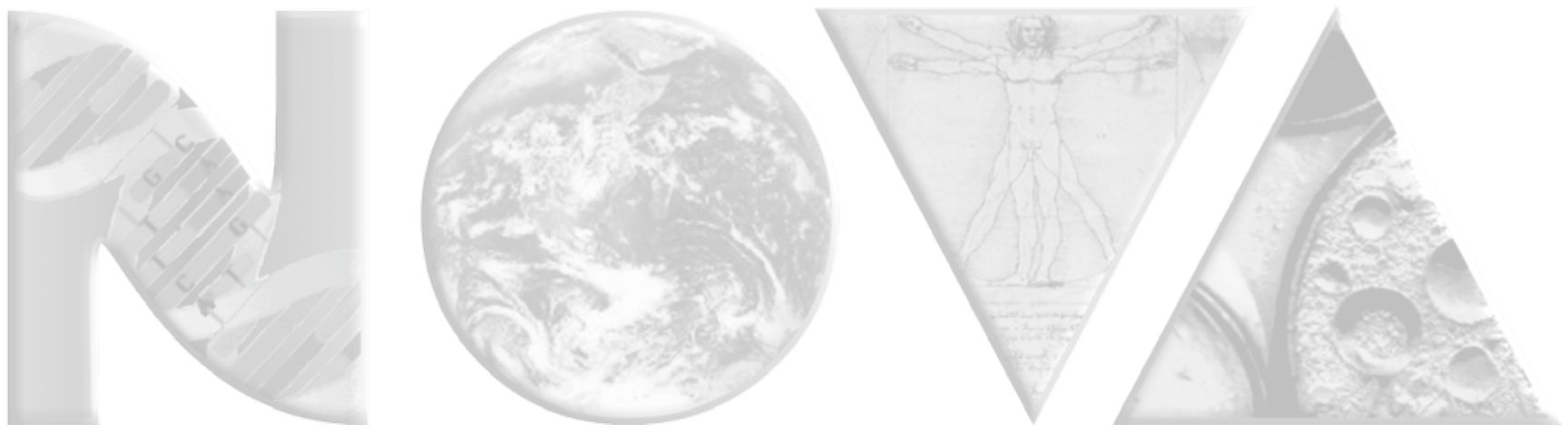